\title{
Harmonic Solutions for the Reflection and Refraction of Finite- Amplitude Acoustic Waves in Two Fluids
}

\author{
Xiao-Mei ZHENG ${ }^{b}$, Xin-Wu ZENGa* \\ Academy of Ocean Science and Engineering, National University of Defense Technology, \\ Changsha 410072, People's Republic of China

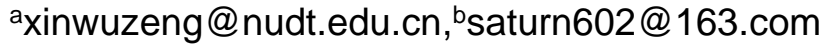

Keywords: Acoustic waves, Finite-amplitude, Harmonic solutions, Fluid interface.

\begin{abstract}
This paper deals with the reflection and refraction of finite-amplitude acoustic waves obliquely incidents to a plane interface between two lossless fluids. The study is based on the second-order harmonic wave equations in term of displacement potential in Lagrangian coordinate system. Variable parameter separation method is used to get the special solutions of the wave equations. Source conditions and boundary conditions are applied to determine the parameters in the special solutions. Results show that the amplitudes of the incident, the reflected and the transmitted harmonic waves all depend on coordinates $a$ and $b$. The solution of incident fluid is composed by three parts: the first part corresponds to the nonlinear interactions of the first acoustic field, with amplitude stimulating along coordinates $a$ and $b$; the second part corresponds to the linear reflection of the second incident wave on the interface and it remains constant when propagation; the third part induced by the interaction between the first incident and reflected waves propagates parallel to the interface with constant amplitude. The special solutions are applicable to wave incidence with any incident angle, not only $0^{\circ}$ and $90^{\circ}$ as mentioned in previous research.
\end{abstract}

\section{Introduction}

Despite precious researches[1,2] have been made last century, the problem of the reflection and transmission of finite-amplitude acoustic waves in fluids has no determined theory as in linear acoustic, i.e. Snell's law and the law of specular reflection. Previous methods are quite different with each other. For example, Van and Breazeale[2,3] dealt with the reflection of finite-amplitude ultrasonic waves by assuming each Fourier component of the incident signal to reflect independently. Nonlinear interaction between the incident and reflected components wasn't considered in their research. Based on the perturbation method, Cotaras[4] derived the second-order harmonic wave equations in term of velocity potential in Euler coordinate system. Nonlinear interaction is indicated by the nonlinear terms of those equations. Although qualities that depend on the derivatives of the second-order velocity potentials may be obtained by numerical calculation, closed form solutions for the second-order velocity potentials have not been obtained. Qian[5,6,7] derived the second-order harmonic wave equations in term of displacement potential in the Lagrangian coordinate system. He proposed a method to solve harmonic wave equations, and got the special solutions. However, these solutions seem to be the special cases of normal incidence or horizontal incidence.

In this paper, we propose a method to get the special solution of the harmonic displacement potential. Use source conditions and boundary conditions to determine the constant parameters in the expressions. The physical significance of special solution is analyzed in detail.

\section{Nonlinear Reflection and Refraction}

\section{Harmonic Wave Equations}

To begin with, the Lagrangian fluid dynamic equations for finite-amplitude acoustic waves in lossless fluid are: 


$$
\begin{aligned}
& \left(\begin{array}{cc}
1+\xi_{a} & \eta_{a} \\
\xi_{b} & 1+\eta_{b}
\end{array}\right)\left(\begin{array}{l}
\xi_{t t} \\
\eta_{t t}
\end{array}\right)=-\frac{1}{\rho}\left(\begin{array}{c}
P_{a} \\
P_{b}
\end{array}\right) \\
& \rho_{0}=\rho\left|\begin{array}{cc}
1+\xi_{a} & \eta_{a} \\
\xi_{b} & 1+\eta_{b}
\end{array}\right| \\
& \left\{\begin{array}{l}
P=P_{0}\left(\frac{\rho}{\rho_{0}}\right)^{\gamma} \text { for } \text { gas } \\
P=P_{0}+A\left(\frac{\rho-\rho_{0}}{\rho_{0}}\right)+\frac{B}{2}\left(\frac{\rho-\rho_{0}}{\rho_{0}}\right)^{2}+\cdots \text { for liquid }
\end{array}\right.
\end{aligned}
$$

where $a$ and $b$ are Lagrangian coordinate variables, $\xi$ and $\eta$ are displacements components. $P$ is pressure, $\rho$ is density. Subscript " 0 " indicate the equilibrium state, while subscripts $a, b$ or $t$ denote partial derivatives with respect to spatial variables $a, b$, or to time variable $t$, respectively. And $\gamma$ is the ratio of specific heat. $A$ and $B$ are the first and the second coefficients of the Taylor series expansion in the pressure-density relation in liquid.

Define a displacement potential $\Phi(a, b, t)$ which satisfies following equations

$$
\partial \Phi / \partial a=\xi, \partial \Phi / \partial b=\eta
$$

Use perturbation method to express $\Phi$ in series expression.

$$
\Phi=\Phi^{(1)}+\Phi^{(2)}+\cdots
$$

Substituting series expression of $\Phi(a, b, t)$ which has left over series higher than second order into Eq.1- Eq.3 yields the primary $(\mathrm{O}(1))$ wave equation and the second-order harmonic $(\mathrm{O}(2))$ wave equation.

$$
\begin{aligned}
\square^{2} \Phi^{(1)} & =\left(\Phi_{a a}^{(1)}+\Phi_{b b}^{(1)}\right)-c_{0}^{-2} \Phi_{t t}^{(1)}=0 \\
\square^{2} \Phi^{(2)} & =\frac{3}{2}\left(\Phi_{a b}^{(1)}\right)^{2}+\frac{y_{1}+y_{2}}{2}+(2 \beta-1) \Phi_{a a}^{(1)} \Phi_{b b}^{(1)} \\
& +\left(\beta-\frac{1}{4}\right)\left[\left(\Phi_{a a}^{(1)}\right)^{2}+\left(\Phi_{b b}^{(1)}\right)^{2}\right]
\end{aligned}
$$

where $\square^{2}=\nabla^{2}-\left(c_{0}\right)^{-2} \partial^{2} / \partial^{2} t$ is the d'Alembertian notation, $c_{0}$ is the small-signal sound speed, $\beta$ is the nonlinear parameter of material. $c_{0}$ and $\beta$ are defined by Eq.3.

$$
\begin{gathered}
c_{0}^{2}=\gamma P_{0} / \rho_{0}, \beta=(\gamma+1) / 2 \quad \text { for ideal gas } \\
c_{0}^{2}=A / \rho_{0}, \beta=1+B / 2 A \quad \text { for liquid }
\end{gathered}
$$

$y_{1}, y_{2}[5]$ are defined as:

$$
\begin{aligned}
& y_{1}=\int\left(\Phi_{a b}^{(1)} \Phi_{b b b}^{(1)}-\Phi_{b b}^{(1)} \Phi_{a a a}^{(1)}\right) \mathrm{d} a \\
& y_{2}=\int\left(\Phi_{a b}^{(1)} \Phi_{a a a}^{(1)}-\Phi_{a a}^{(1)} \Phi_{b b b}^{(1)}\right) \mathrm{d} b
\end{aligned}
$$

The relationship between the pressure and the displacement potential[5]

$$
\begin{gathered}
p^{(1)}=-\rho_{0} \frac{\partial^{2} \Phi^{(1)}}{\partial t^{2}} \\
p^{(2)}=-\rho_{0}\left[\frac{\partial^{2} \Phi^{(2)}}{\partial t^{2}}+\int\left(\frac{\partial \Phi^{(1)}}{\partial a \partial b} \frac{\partial^{3} \Phi^{(1)}}{\partial t^{2} \partial b}-\frac{\partial^{2} \Phi_{1}^{i}}{\partial b^{2}} \frac{\partial^{3} \Phi^{(1)}}{\partial t^{2} \partial a}\right) \mathrm{d} a\right] \\
=-\rho_{0}\left[\frac{\partial^{2} \Phi^{(2)}}{\partial t^{2}}+\int\left(\frac{\partial^{2} \Phi^{(1)}}{\partial a \partial b} \frac{\partial^{3} \Phi^{(1)}}{\partial t^{2} \partial a}-\frac{\partial^{2} \Phi^{(1)}}{\partial a^{2}} \frac{\partial^{3} \Phi^{(1)}}{\partial t^{2} \partial b}\right) \mathrm{d} b\right]
\end{gathered}
$$




\section{Source Conditions and Boundary Conditions}

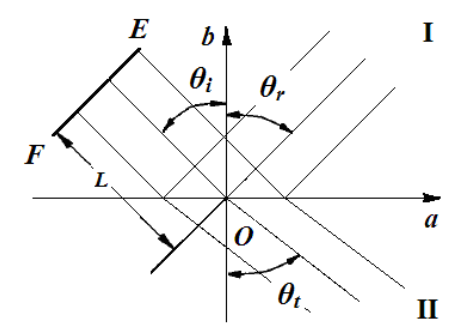

Figure 1. Reflection and refraction on the interface

Consider finite amplitude plane wave $P=P_{0} e^{-j(\omega t-k 1 a+k 2 b-k L)}\left(E F: b \cos \theta_{i}-a \sin \theta_{i}=L\right)$ obliquely incident to the interface between fluid I and II, where reflection and refraction will occur (see Fig.1). Suppose $\theta_{i}, \theta^{\prime}{ }_{i}$ and $\theta_{t}$ are the incident angle, the reflection angle and the refraction angle, respectively. The $\mathrm{O}(1)$ and the $\mathrm{O}(2)$ acoustic field should satisfy: the pressure and the normal component of the particle velocity are continuous at the interface.

$$
\begin{gathered}
\left.P_{\mathrm{I}}^{(1)}\right|_{b=0}=\left.P_{\mathrm{II}}^{(1)}\right|_{b=0},\left.P_{\mathrm{I}}^{(2)}\right|_{b=0}=\left.P_{\mathrm{II}}^{(2)}\right|_{b=0} \\
\left.\frac{\partial^{2} \Phi_{\mathrm{I}}^{(1)}}{\partial t \partial b}\right|_{b=0}=\left.\frac{\partial^{2} \Phi_{\mathrm{I}}^{(1)}}{\partial t \partial b}\right|_{b=0},\left.\frac{\partial^{2} \Phi_{\mathrm{I}}^{(2)}}{\partial t \partial b}\right|_{b=0}=\left.\frac{\partial^{2} \Phi_{\mathrm{II}}^{(2)}}{\partial t \partial b}\right|_{b=0}
\end{gathered}
$$

So the $\mathrm{O}(1)$ acoustic fields are

$$
\begin{array}{r}
P_{\mathrm{I}}^{(1)}=P_{0}\left(e^{-j\left(\omega t+\delta_{1}\right)}+V e^{-j\left(\omega t+\delta_{2}\right)}\right), P_{\mathrm{II}}^{(1)}=P_{0} W e^{-j\left(\omega t+\delta_{3}\right)} \\
\Phi_{\mathrm{I}}^{(1)}=\Lambda\left(e^{-j\left(\omega t+\delta_{1}\right)}+V e^{-j\left(\omega t+\delta_{2}\right)}\right), \Phi_{\mathrm{II}}^{(1)}=\Lambda \frac{W}{m} e^{-j\left(\omega t+\delta_{3}\right)}
\end{array}
$$

where $\Lambda=P_{0} / \rho_{1} \omega^{2}, \quad k_{1}\left(=k \sin \theta_{i}\right)$ and $k_{2}\left(=k \cos \theta_{i}\right)$ are two components of wave number $k\left(=\omega / c_{1}\right)$ in fluid I, $l_{1}\left(=l \sin \theta_{t}\right)$ and $l_{2}\left(=l \cos \theta_{t}\right)$ are components of wave number $l\left(=\omega / c_{2}\right)$ in fluid II. $\delta_{1}=-k_{1} a+k_{2} b$ $k L, \delta_{2}=-k_{1} a-k_{2} b-k L, \delta_{3}=-l_{1} a+l_{2} b-k L$. Reflection coefficient $V$ and refraction coefficient $W$ are

$$
V=\frac{m \cos \theta_{i}-n \cos \theta_{t}}{m \cos \theta_{i}+n \cos \theta_{t}}, W=\frac{2 m \cos \theta_{i}}{m \cos \theta_{i}+n \cos \theta_{t}}
$$

where $m=\rho_{2} / \rho_{1}, n=c_{1} / c_{2}$. Substituting Eq.14 into Eq.7 leads to the $\mathrm{O}(2)$ wave equations in fluid I and II

$$
\begin{gathered}
\square^{2} \Phi_{\mathrm{I}}^{(2)}=4 \Lambda^{2}\left[\alpha_{2} e^{-2 j k_{2} b}+\alpha_{2} V^{2} e^{2 j k_{2} b}+\alpha_{1} V\right] e^{-2 j\left(\omega t-k_{1} a-k L\right)} \\
\alpha_{1}=\frac{1}{2}\left[\left(\beta_{1}-1\right) k^{4}+\left(k_{1}^{4}-k_{1}^{2} k_{2}^{2}\right)\right], \alpha_{2}=\beta_{1} k^{4} / 4 \\
\square^{2} \Phi_{\mathrm{II}}^{(2)}=4 \alpha_{3}(W / m)^{2} \Lambda^{2} e^{-2 j\left(\omega t-l a-l_{2} b-k L\right)}, \alpha_{3}=\beta_{2} l^{4} / 4
\end{gathered}
$$

\section{Harmonic Solutions}

Theoretically, the total solution of Eq.7 consists of two parts: the special solution and the general solution of the corresponding homogeneous equation. General solution is easy to get. To obtain the special solution, divide Eq.7 into three parts

$$
\begin{aligned}
& \square^{2} \Phi_{\mathrm{I}}^{(2)}=4 \Lambda^{2} \alpha_{2} e^{-2 j k_{2} b} e^{-2 j\left(\omega t-k_{1} a-k L\right)} \\
& \square^{2} \Phi_{\mathrm{I}}^{(2)}=4 \Lambda^{2} \alpha_{2} V^{2} e^{2 j k_{2} b} e^{-2 j\left(\omega t-k_{1} a-k L\right)}
\end{aligned}
$$




$$
\square^{2} \Phi_{I}^{(2)}=4 \Lambda^{2} \alpha_{1} V e^{-2 j\left(\omega t-k_{1} a-k L\right)}
$$

which represent the self-action of the $\mathrm{O}(1)$ incident wave, the self-action of the $\mathrm{O}(1)$ reflected wave and the interaction of the $\mathrm{O}(1)$ incident and reflected wave, respectively. And Eq.19 represents the self-action of the $\mathrm{O}(1)$ refracted wave.

If $\varphi_{1}, \varphi_{2}, \varphi_{3}$ are the special solutions of Eq.20-Eq.22, respectively, then $\varphi_{1}+\varphi_{2}+\varphi_{3}$ should be a special solution of Eq.18.

Apply segregation variable method to solve these four equations Eq.19-22. For example, suppose the special solution of Eq.20 $\varphi_{1}$ to be

$$
\varphi_{1}=\varphi_{11}(a) e^{-2 j k_{2} b}+\varphi_{12}(b) e^{2 j k_{1} a}
$$

where the item $e^{-j(\omega t-k L)}$ has been dropped for simplicity. Substituting Eq.23 into Eq.20 leads to an ordinary differential equation

$$
\begin{array}{r}
\left(\frac{\mathrm{d}^{2} \varphi_{11}}{\mathrm{~d} a^{2}}+4 k_{1}^{2} \varphi_{11}\right) e^{-2 j k_{2} b}+\left(\frac{\mathrm{d}^{2} \varphi_{12}}{\mathrm{~d} b^{2}}+4 k_{2}^{2} \varphi_{12}\right) e^{2 j k_{1} a} \\
=4 \Lambda^{2} \alpha_{2} e^{-2 j k_{2} b} e^{2 j k_{1} a}
\end{array}
$$

Divide Eq.24 to two equations, drop the item $e^{-2 j k 2 b}$ in the first equation and $e^{-2 j k 1 a}$ in the second equation, and obtain the following simplified equations

$$
\begin{gathered}
\frac{\mathrm{d}^{2} \varphi_{11}}{\mathrm{~d} a^{2}}+4 k_{1}^{2} \varphi_{11}=4 \Lambda^{2} m_{1} \alpha_{2} e^{2 j k_{1} a} \\
\frac{\mathrm{d}^{2} \varphi_{12}}{\mathrm{~d} b^{2}}+4 k_{2}^{2} \varphi_{12}=4 \Lambda^{2}\left(1-m_{1}\right) \alpha_{2} e^{-2 j k_{2} b}
\end{gathered}
$$

where $m_{1}$ is an important dimensionless parameter can be determined by source condition. Then work out $\varphi_{11}, \varphi_{12}$

$$
\left\{\begin{array}{l}
\varphi_{11}=m_{1} \alpha_{2}^{\prime}\left(\frac{a}{j k_{1}}+\frac{1}{4 k_{1}^{2}}\right) e^{2 j k_{1} a} \\
\varphi_{12}=\left(1-m_{1}\right) \alpha_{2}^{\prime}\left(-\frac{b}{j k_{2}}+\frac{1}{4 k_{2}^{2}}\right) e^{-2 j k_{2} b}
\end{array}\right.
$$

consequently obtain the special solution of Eq.20

$$
\begin{aligned}
& \varphi_{1}=\Lambda^{2} \alpha_{2} \\
& {\left[m_{1}\left(\frac{a}{j k_{1}}+\frac{1}{4 k_{1}^{2}}\right)+\left(1-m_{1}\right)\left(-\frac{b}{j k_{2}}+\frac{1}{4 k_{2}^{2}}\right)\right] e^{2 j k_{1} a} e^{-2 j k_{2} b}}
\end{aligned}
$$

Other solutions of Eq.19, Eq.21, Eq.22 can been derived in the same way. The right side of Eq.22 only depends on coordinate $a$, whose special solution is

$$
\varphi_{3}=\Lambda^{2} \frac{\alpha_{1} V}{k_{2}^{2}} e^{2 j k_{1} a}
$$

Therefore the $\mathrm{O}(2)$ displacement potentials in fluid I and II are obtained

$$
\begin{gathered}
\Phi_{\mathrm{I}}^{(2)}=\Lambda^{2} e^{2 j\left(k_{1} a+k L-\omega t\right)}\left\{\alpha_{2}\left(\frac{m_{1}}{j k_{1}} a-\frac{1-m_{1}}{j k_{2}} b+Q_{1}\right) e^{-2 j k_{2} b}\right. \\
\left.+\alpha_{2} V^{2}\left(\frac{m_{2}}{j k_{1}} a+\frac{1-m_{2}}{j k_{2}} b+Q_{2}\right) e^{2 j k_{2} b}+\frac{\alpha_{1} V}{k_{2}^{2}}\right\}
\end{gathered}
$$




$$
\Phi_{\mathrm{II}}^{(2)}=\Lambda^{2} \alpha_{3}\left(\frac{W}{m}\right)^{2}\left(\frac{m_{3}}{j l_{1}} a-\frac{1-m_{3}}{j l_{2}} b+Q_{3}\right) e^{2 j\left(l_{1} a-l_{2} b+k L-\omega t\right)}
$$

where $Q_{1}, Q_{2}, Q_{3}$ with relation to $m_{1}, m_{2}, m_{3}$ are constants to be determined by source conditions and boundary conditions.

The $\mathrm{O}(2)$ potential-pressure relations can be derived by Eq.11, Eq.12

$$
\begin{gathered}
P_{\mathrm{I}}^{(2)}=4 \rho_{\mathrm{l}} \omega^{2} \Phi_{\mathrm{I}}^{(2)}+2 \rho_{1} k_{2}^{2} \omega^{2} V \Lambda^{2} e^{2 j\left(k_{1} a+k L-\omega t\right)} \\
P_{\mathrm{II}}^{(2)}=4 \rho_{2} \omega^{2} \Phi_{\mathrm{II}}^{(2)}
\end{gathered}
$$

where the second term in Eq.32 is due to the interaction between the $\mathrm{O}(1)$ incident wave and reflected wave.

Using Eq.32 and Eq.33, the $\mathrm{O}(2)$ pressures are expressed

$$
\begin{aligned}
P_{\mathrm{I}}^{(2)} & =\frac{4 P_{0}^{2}}{\rho_{1} \omega^{2}}\left\{\alpha_{2}\left(\frac{m_{1}}{j k_{1}} a-\frac{1-m_{1}}{j k_{2}} b+Q_{1}\right) e^{-2 j k_{2} b}\right. \\
& +\alpha_{2} V^{2}\left(\frac{m_{2}}{j k_{1}} a+\frac{1-m_{2}}{j k_{2}} b+Q_{2}\right) e^{2 j k_{2} b} \\
& \left.+V\left(\frac{\alpha_{1}}{k_{2}^{2}}+\frac{k_{2}^{2}}{2}\right)\right\} e^{2 j\left(k_{1} a+k L-\omega t\right)} \\
P_{\mathrm{II}}^{(2)}= & \frac{4 P_{0}^{2}}{\rho_{2} \omega^{2}} \alpha_{3} W^{2}\left(\frac{m_{3}}{j l_{1}} a-\frac{1-m_{3}}{j l_{2}} b+Q_{3}\right) e^{2 j\left(l_{1} a-l_{2} b+k L-\omega t\right)}
\end{aligned}
$$

Note that there are only incident components near the source. Moreover, no self-action of the primary wave has occurred at the source, that is to say the $\mathrm{O}(2)$ pressure equals to zero

$$
\begin{aligned}
P_{\mathrm{I}}^{(2)} & =\frac{4 P_{0}^{2} \alpha_{2}}{\rho_{1} \omega^{2}}\left(\frac{m_{1}}{j k_{1}} a-\frac{1-m_{1}}{j k_{2}} b+Q_{1}\right) e^{2 j\left(k_{1} a-k_{2} b+k L-\omega t\right)} \\
& =0 \quad \text { on } b \cos \theta_{i}-a \sin \theta_{i}=L
\end{aligned}
$$

which leads to the results

$$
m_{1}=\sin ^{2} \theta_{i}
$$

Once the incident angle $\theta_{i}$ is determined, $m_{1}$ will be determined accordingly. Eq.28 is written as

$$
\varphi_{1}=\Lambda^{2} \alpha_{2}\left[\frac{\sin ^{2} \theta_{i}}{j k_{1}} a-\frac{\cos ^{2} \theta_{i}}{j k_{2}} b+\frac{1}{2 k^{2}}\right] e^{2 j\left(k_{1} a-k_{2} b\right)}
$$

Other parameters, $m_{2}, m_{3}, Q_{1}, Q_{2}, Q_{3}$, can been determined by boundary conditions. The detailed derivation is omitted in this paper.

\section{Discussion}

Qian[7] has considered the special solution of Eq.20' kind to be

$$
\varphi_{1}=\Lambda^{2} \alpha_{2}\left(A_{1} a+C_{1}\right) e^{2 j\left(k_{1} a-k_{2} b+k L-\omega t\right)}
$$

or

$$
\varphi_{1}=\Lambda^{2} \alpha_{2}\left(B_{1} b+C_{1}\right) e^{2 j\left(k_{1} a-k_{2} b+k L-\omega t\right)}
$$

meaning that the nonlinear situmulation only occur along coordinate $a$ or $b$, not both. Note that it is just the condition of incident angle $\theta_{i}=0^{\circ}$ or $90^{\circ}$ in Eq. 38 as 


$$
\begin{gathered}
\varphi_{1}=\Lambda^{2} \alpha_{2}\left[-\frac{1}{j k_{2}} b+\frac{1}{2 k^{2}}\right] e^{2 j\left(k_{1} a-k_{2} b\right)} \\
\varphi_{1}=\Lambda^{2} \alpha_{2}\left[\frac{1}{j k_{1}} a+\frac{1}{2 k^{2}}\right] e^{2 j\left(k_{1} a-k_{2} b\right)}
\end{gathered}
$$

For the solution of other incident angle, we should refer to Eq.38.

The $\mathrm{O}(2)$ displacement potential in fluid I, Eq. (30), is composed by three parts:

(1) the first part that doesn't consist $V$ or $V^{2}$ corresponds the self-action of the $\mathrm{O}(1)$ incident wave;

(2) the second part consists $V^{2}$ corresponds to the self-action of the $\mathrm{O}(1)$ reflected wave;

(3) the third part consists $V$ corresponds the self-action of the $\mathrm{O}(1)$ incident wave and the $\mathrm{O}(1)$ reflected wave. It propagates parallel to the interface with constant amplitude. The amplitudes of precious two parts depends on coordinates $a$ and $b$ and grows while propagation.

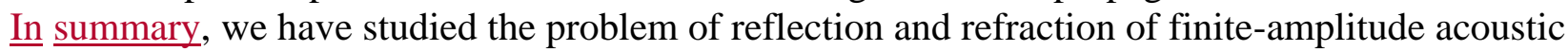
wave at the fluid-fluid plane interface, and analyzed the harmonic solutions in details. The special solutions are applicable to wave incidence situation with any incident angle, not only $0^{\circ}$ and $90^{\circ}$ as mentioned in previous research. The derivation and the results in this study make some supplement to the theory of nonlinear wave reflection and refraction.

\section{References}

[1] D. T. Blackstock, Proceedings of the $3^{\text {rd }}$ International Conference of Acoustic, 309, 1959.

[2] L. A. Van Buren, M. A. Breazeale, J. Acoust. Soc. Am. 44, 1014-1020 (1968).

[3] L. A. Van Buren, M. A. Breazeale, J. Acoust. Soc. Am. 44, 1020-1027(1968).

[4] F. D. Coteras, Reflection and refraction of finite amplitude acoustic waves at a fluid-fluid interface, (1988) .

[5] Z. W. Qian, Scientia sinica (Series A) 6, 492-501 (1982) .

[6] Z. W. Qian, Acta Physica Sinca. 42, 949-953 (1993).

[7] Z. W. Qian, X. Y. Zheng, Chin. Phys. Lett., 6, 305-308, (1989). 